\title{
Multi-use offshore platform configurations in the scope of the FP7 TROPOS Project
}

E. Quevedo, M. Cartón, E. Delory, A. Castro, J. Hernández and O. Llinás

Oceanic Platform of the Canary Islands Carretera de Taliarte s/n, 35200 Telde, Spain e-mail: tropos@plocan.eu

\section{J. de Lara}

Polytechnic University of Madrid Avenida Ramiro de Maeztu 7, 280840 Madrid, Spain e-mail: jose.delara@upm.es

N. Papandroulakis, P. Anastasiadis

Institute of Marine Biology, Biotechnology and Aquaculture, Hellenic Centre for Maritime Research

AquaLabs, Thalassocosmos, Crete, Greece e-mail:npap@hcmr.gr

\begin{abstract}
The FP7 funded TROPOS project approach is to develop a modular multi-use platform for use in deep waters, with a focus on the Mediterranean, tropical and sub-tropical regions. In this paper, three different platforms configurations, which have been designed to show the synergies and compatibilities among the platform uses of Transport, Energy, Aquaculture and Leisure - are presented.
\end{abstract}

Keywords- fixed structure, multi-purpose, multi-use, offshore, platform.

\section{INTRODUCTION}

The analysis, design and construction of offshore structures is arguably one of the most demanding set of tasks faced by the engineering profession. Over and above the usual conditions and situations met by land-based structures, offshore structures have the added complication of being placed in an ocean environment where hydrodynamic interaction effects and dynamic response become major considerations in their design [1].

Offshore platforms can be broadly categorized in two types (some of these platforms are shown in Figure 1):

- Fixed structures which extend to the seabed: Steel Jacket, Concrete Gravity Structure and Compliant Tower.

This work has been supported by the FP7 TROPOS Project. Funding for the TROPOS project (GA No. 288192) was received from the EU Commission as part of the 7th Framework programme "The Ocean of Tomorrow" theme, OCEAN.2011-1: Multi-use Offshore Platforms. The TROPOS project is solely responsible for this publication. It does not represent the opinion of the EU.

\author{
J. Bard \\ Fraunhofer Institute for Wind Energy and Energy Systems \\ Technology IWES - \\ Königstor 59, 34119 Kassel, Germany \\ e-mail: jochen.bard@iwes.fraunhofer.de
}

H. Jeffrey and D. Ingram

The University of Edinburgh -

Old College, South Bridge, eh8 9yl Edinburgh, UK

e-mail: henry.jeffrey@ed.ac.uk

J. Wesnigk

Universität Bremen MARUM

PO Box 330 440, Bremen, Germany

e-mail: jwesnigk@nwv-meeresforschung.de

for the TROPOS Consortium

- Structures which float near the water surface: Tension Leg Platforms (TLPs), Semisubmersible, Spar and Floating, Production, Storage and Offloading (FPSO) ship shaped vessel.

The evolution of the offshore platforms has made possible to think in other possibilities parallel to the oil exploitation. At the same time, the European Marine Spatial Planning advises to share sites, infrastructure and costs in these diverse activities [2]. Aware of this reality, the European Union launched "The Ocean of Tomorrow" call for proposals for multi-use offshore platforms. Through this topic, the EU has provided the scientific and entrepreneurial community with a total funding of 14 million Euros, for up to three projects dedicated to the design of multi-use offshore Platforms [3]. Three projects were selected in the scope of this call - H2OCEAN [4], MERMAID [5] and TROPOS [6].

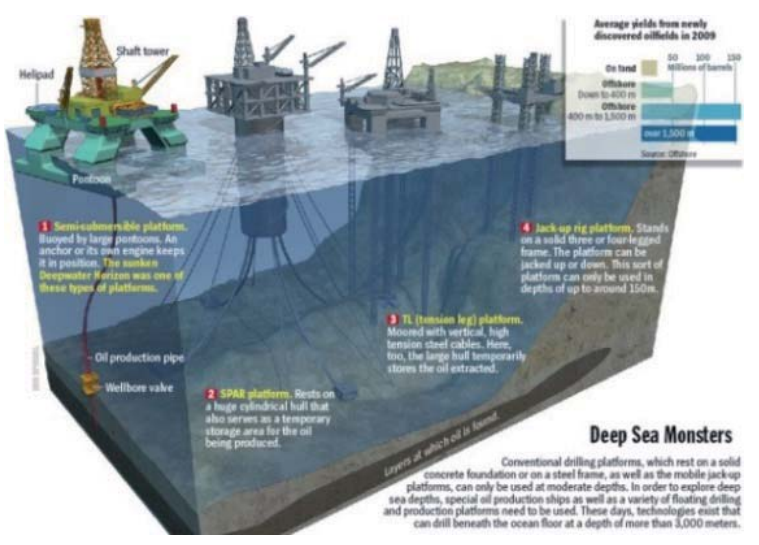

Fig. 1. Offshore Oil and Gas platforms evolution [7] 
The FP7 (7th Framework Programme) funded TROPOS project approach is to develop a modular multi-use platform for use in deep waters, with focus on Mediterranean, tropical and sub-tropical regions.

\section{TROPOS PROJECT APPROACH}

The TROPOS project approach consists in the development of a multi-use platform system integrating a range of activities from the transport, energy, aquaculture and leisure sectors, which will help to overcome challenges in each of these sectors, primarily, among other benefits, the shared use of a common infrastructure.

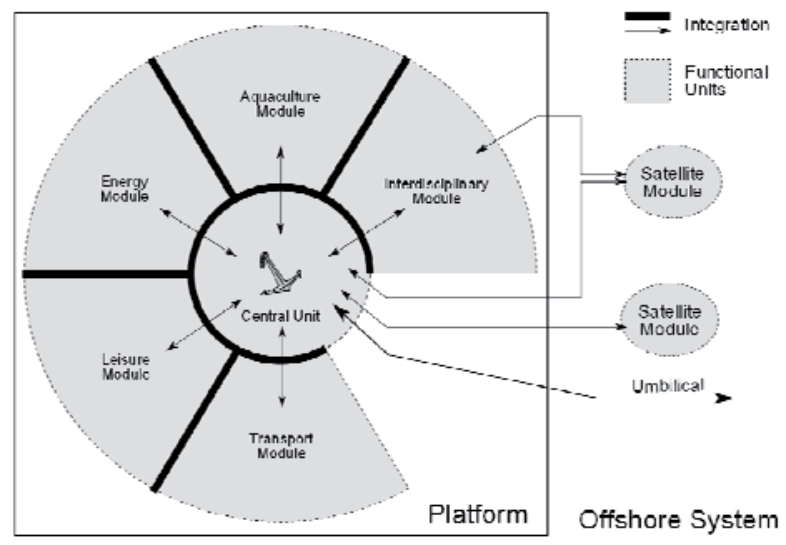

Fig. 2. Multi-use offshore platform functional components [8]

A key to the modular development in the TROPOS approach stems from the old aphorism: "Think globally, act locally". Global design generally implies the development of standard solutions; however, the diversity of regional scenarios requires a degree of flexibility and adaptability. In TROPOS flexibility is achieved through the use of modular units, where different types of modules can be combined to fit the unique environmental and socio-economic conditions in each area.

\section{TROPOS PLATFORM CONCEPTS}

Aiming to enhance the potential and increase the added value of integrating the four components of the platform, namely Transport, Energy, Aquaculture and Leisure (the socalled TEAL), in TROPOS an Interdisciplinary Cohesion Subcommittee (ICS) is operated. In this frame, three different platforms configurations (concepts) have been designed in order to form an initial graphic idea of the synergies and compatibilities among components:

- Industrial Complex Concept.

- Green \& Blue Concept.

- Leisure Island Concept.

These concepts are used as examples in order to discuss proposed methodologies. This is facilitating the project progress and improving the establishment of methodologies along all TEAL aspects. The proposed concepts do not replace the detailed investigations and analysis which are currently being carried out under the several work package tasks in the project and which will deliver their own detailed concept studies and results.

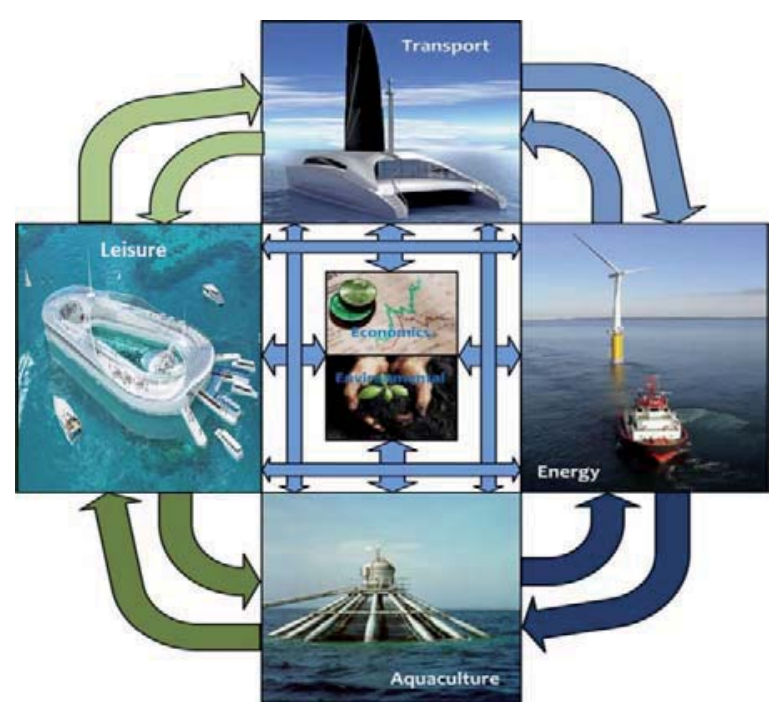

Fig. 3. Four TEAL (Transport, Energy, Aquaculture and Leisure) components are studied in regard to their combined technical and economic viability and environmental impacts

Therefore the three different platform configurations (concepts) presented in this paper form an initial basis in the TROPOS project to begin the engineering design of the platform. This process will consider other project inputs related to the constraints and the resource assessment for the TEAL components and will include thorough economic, environmental and socio-economic assessment of the potentially significant impacts in relation to selected conceptlocation combinations.

\section{A. Industrial Complex Concept}

This concept consists of a modular industrial type of a far offshore structure which is focusing on transport and energy related aspects while it still has aquaculture and some leisure related aspects. It implements a large floating offshore port with infrastructure relating to repair and maintenance of large ships, including floating docks, the required lifting capacities, different workshops and material storage and an offshore terminal for handling of dangerous goods such as oil and gas.

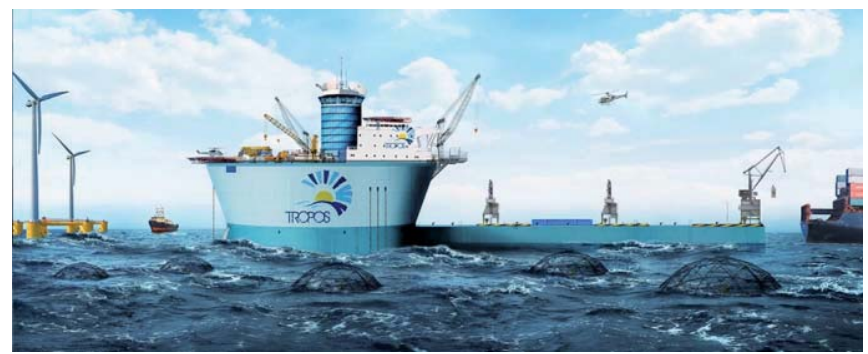

Fig. 4. Industrial Complex Concept Representation [9]

According to the conclusions presented in the Project Deliverable 5.1 [10], the industrial complex design seems to be 
quite adequate for multidisciplinarity since the main influences that the TROPOS platform would have on each of the TEA (Transport, Energy and Aquaculture) industries are as follows:

- The TROPOS platform offers a unique scenario for reducing operation and maintenance costs in the marine transport, energy and aquaculture sectors.

- Further investment and R\&D in stable, floating platforms and structures will facilitate the development of floating wind turbine structures.

- The implementation of photovoltaics and solar heating / cooling systems can act to decrease the overall cost of the technology.

- The implementation of OTEC (Ocean Thermal Energy Conversion), where possible, within the platform structure can act to decrease construction costs of a plant.

- Although tidal energy devices and resources are generally located in relatively shallow waters, the platform could act as a servicing hub for devices, or act as a satellite platform which contains the devices, thus promoting the innovation of tidal energy device technologies.

- There is currently a high cost of electricity for remote and isolated regions, the inclusion of a wave energy device can work toward reducing this cost, where favourable wave resources exist.

- The construction of a TROPOS platform will aid the development of offshore aquaculture technologies and will allow exploitation of deeper water fishery resources.

- The shipping industry would benefit from the construction of ship repairing facilities at medium sized floating harbours (these can also be used as fish processing plants).

\section{$\underline{\text { A.1.- Transport components }}$}

It realises a large floating offshore port with infrastructure towards repair and maintenance of large ships including floating docks, the required lifting capacities, different workshops and material storage; an offshore terminal for handling of dangerous goods such as oil and gas.

Depending on the location of the site it might support the offshore mining industry with infrastructure related to handling equipment as well as personnel. It provides accommodation for these industries work forces. Ships are provided with fuels as well as electrical energy during their stay in the dock. Fresh water and food supply is provided to the ships if appropriate e.g. to cruise ships (even though they seem to be mostly selfsufficient nowadays).

\section{A.2.- Energy components}

The concept includes very significant renewable energy production facilities in the form of wind turbines and wave energy devices. Where the structure of the port allows, fixed wind turbines are installed without compromising the operation of cranes etc. In addition a floating offshore wind farm is assembled around the port, leaving enough clearance for a safe access of large ships to the port. The electrical energy generated by the wind turbines directly supplies the electrical power consumers of the entire facility. In addition excess energy is used to produce hydrogen and gases or liquid fuels (ammonia, Power2Gas as in the form of CNG or LNG, methanol and other hydrocarbons). For the P2G installation $\mathrm{CO} 2$ is required in large quantities which are provided to the site by means of tankers.

The produced fuels are used as a means of energy storage as well as to ensure the power supply of the facilities at all times. This is realised by using conventional cogeneration technologies (e.g. internal combustion engines) or alternatively advanced energy conversion technologies based on hydrogen (e.g. fuel cells) which is produced from water electrolysis. For this purpose water desalination and purification systems are installed. Solar thermal as well as PV systems can be added to the system if appropriate to efficiently provide hot water as well as additional power during phases of low wind speeds. The accommodation of the energy technology related service personnel is realised on the platform. The port infrastructure allows for major repair work related even to the large floating WTs.

\section{A.3.- Aquaculture components}

The concept provides energy in the form of electricity as well as heat (and also cooling if needed), freshwater and CO2. In case that transport activity includes infrastructure towards repair and maintenance of large ships, different workshops and material storage, an offshore terminal for handling of dangerous goods such as oil and gas, then a potential conflict with the aquaculture module could exist and in particular for the food oriented cultures. This is because aquaculture requires high water quality which is jeopardized with the above activities. The main advantage for aquaculture to move offshore is that inshore restrictions (environmental, geographical, political) are overcome regarding. In this sense if transport activity is the priority (i.e. the above mentioned restrictions are also present off shore), the aquaculture module will be limited to a minimum scale if the required conditions are met.

Respecting the above limitations, the fish culture unit can be realized in the form of floating/ submerged cages. The location of the cages should be preferably in between the windturbines and taking advantages of their structures, preferably at the leeward side of the platform. Depending on the final design cages may be also located closer to the platform. Feeding will be managed either from the platform or from independent floating silos in the vicinity of the cages. Secondary cultures will be developed for bivalves and/ or macroalgae depending on the location while bottom cultures could be also established (if the depth allows such an activity). The unit, depending on its capacity, could include a processing facility.

The algae culture unit will be based both on natural resources and domestic wastes. In particular, the waste water from the accommodation facilities and partially from ships using the port will be treated at the site and organic waste will be available for specific aquacultures. These could be algae, 
will depend on the type of waste and the biomass produced from this activity will not be for consumption. Bioremediation provides a sustainable solution for treatment of wastewaters from several sources. Algae are mainly applied for nutrient reduction, but algae are also able to metabolize both inorganic carbon and organic carbon at the same time in a mixotrophic cultivation and can provide an advantage for enhanced biomass production. For the platform domestic, vegetable and industrial wastewaters can be applied mainly for the microalgae production. These can be integrated into the port infrastructure as well as separated in some distance. The concept includes very significant renewable energy.

\section{$\underline{\text { A.4.- Leisure components }}$}

Due to the accommodation infrastructure for the workforces, this concept has capacity to host a large number of people. The infrastructure is also ready for external visits (controlled and following strict security measures). Offshore R\&D related accommodation can be offered. Fresh water and to some extent food (e.g. fish and other aqua farming products) are locally available. The waste heat of the electricity generation is used for heating purposes, the waste and waste water is collected and utilised for the algae related production cycles. The facility provides other services, such as a research platform, offers oceanographic and atmospheric measurements, traffic and marine safety services (monitoring as well as rescue facilities).

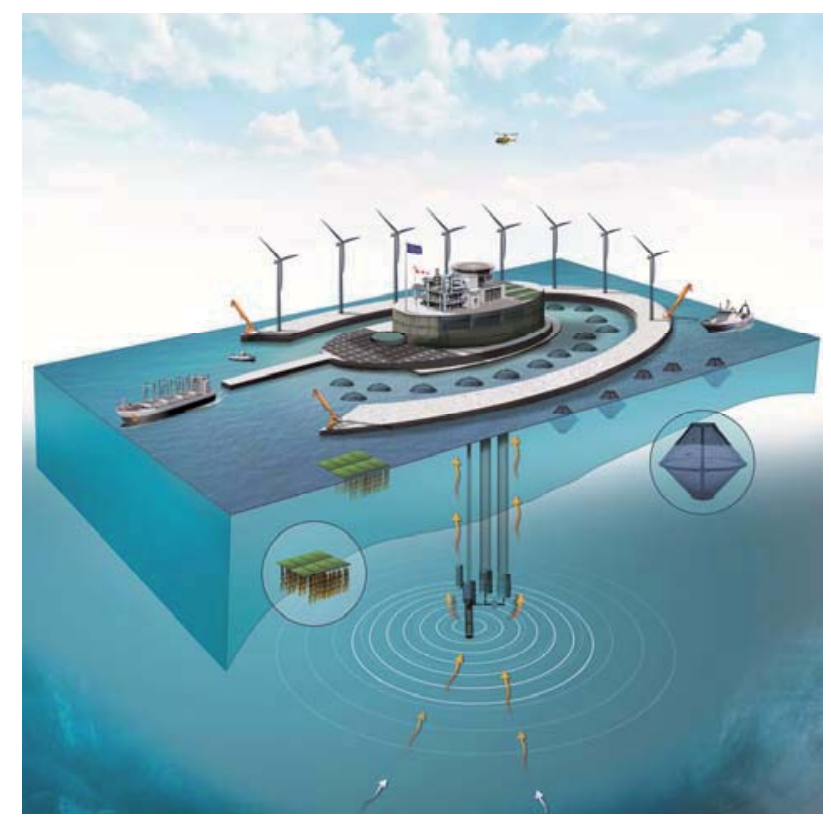

Fig. 5. Green\&Blue Concept Representation [9]

\section{B. Green \& Blue Concept}

The Green \& Blue Concept will be mainly based on the Energy and Aquaculture Components. This concept combines an offshore wind farm and potentially other renewable energies such as wave energy devices or OTEC with different aquaculture installations. It will include different modules and a central part. It will consist of port facilities for small cargo fisheries and also for the logistics of the platform, a Renewable
Energy Hub Collector and an Aquaculture Module including fish (IMTA), algae (micro and macro algae), biomass and hatcheries.

As the concepts for shallow waters are being investigated in great detail within the MERMAID project the focus of the TROPOS "Green \& Blue" concept will be on deep water solutions utilizing floating energy related platforms as part of the mechanical structure. It is expected to integrate aquaculture installations such as floating cages, either into the structure of the floater itself (in the case of semi-submersible platforms) or to use the mooring points and the floating structure as connections for the aquaculture installations. In addition there will be synergies with regard to the energy supply and the maintenance and operation of these combined uses. This concept avoids the combination with other uses such industrial activities which might compromise e.g. the water quality and other crucial aspects of the aquafarming activities.

The main concept is divided into two main sub-concepts:

- Fish aquaculture plus floating offshore wind and wave.

- OTEC plus aquaculture, "sea ranching".

\section{B.1.- Fish aquaculture plus floating offshore wind and wave}

The energy part of this concept consist of a floating wind farm using TLPs, semisubmersibles or spar technology in combination with various wave energy concepts, e.g. small point absorbers or terminator units combined with a TLP or semi-submersible. Such concepts are known from developers such as Principle Power's Wind Wave Float, W2Power from Pelagic and others. Further technical details of such concepts can be found in Project Deliverable 2.1 table 2-1 Multi use offshore platforms and additional details are provide in the Excel tables "Multi-use platform concepts comprising wind energy" as provided by Fraunhofer [10]. Also Spar concepts are possible with more limited options to integrate wave power structurally - however the combination of independent floating devices such as Pelamis or others moored and grid connected with the floating wind farm is possible.

A fish culture module in the form of floating, submerged or drifting cages takes advantage of the facilities that the platform offers, namely energy, protection, anchoring and logistics with regard to installation, maintenance and operation. The module should consist of units (e.g. sea cages) of the appropriate technology that should be selected/ developed to allow operation with high levels of autonomy. The procedure will be to keep the fish, fed with artificial diets, until harvesting them at an appropriate size. Whereas a minimum scale system would only exist of the cages, medium scales would also include a hatchery for on-site juvenile production and a processing unit for on-site processing of the marketable fish. The processing unit will integrate the fishery activity and could include industrial processing facilities of fishery products (e.g. frozen, canned, smoked, cold storage, packaging, etc.) with significant economic benefit to the fishing fleet too.

Secondary cultures, round the cages, will be developed for bivalves and/ or macroalgae depending on the location. 
Additionally, if the location's depth allows such an activity, bottom cultures could be also established.

The algae culture unit will be based on natural resources and domestic wastes and will include:

- Microalgae and macro-algae production.

- Products with high market value (clean/ natural nutrients $+\mathrm{CO} 2$ sources).

- Biofuel/gasification from macro- and microalgae.

- $\mathrm{CO} 2$ fixation from fluegases.

- Microalgae in closed photobioreactors with nutrients from the platforms wastewaters and a mixtotrophic production with carbon source.

- Macroalgae production (applied as wastewater treatment).

The algae unit will be located close to the platform and the biomass will be the feedstock for the energetic conversion as gasification, fermentation and biofuels. Its location should be in the most protected area to avoid significant wave heights and currents. For the high-value products the water-quality, nutrients and CO2-source must be selected more carefully.

\section{B.2 OTEC plus aquaculture, "sea ranching"}

The second approach is related to the potential of bringing Deep Oceanic Water to the surface [10], i.e. the creation of an artificial up welling coupled with the Ocean Thermal Energy Conversion action. The possible applications of DOW and OTEC approaches could be called as "Sea Ranching".

The main energy related component is the OTEC system. This structure could however still have a large wind turbine or wave energy converters integrated structurally or with regard to the mooring and grid connection. The technical details of OTEC and combined concepts are provided in Project Deliverable 2.1 [11]. The key aspect is however the availability of large amounts of deep oceanic water (artificial upwelling) that could be also explored by aquaculture. This would have a beneficial impact on the ecosystem and increase the local natural productivity supported by specific aquaculture installations. Again this minimum solution would provide energy supply and logistics as well as station keeping synergies.

A more advanced variation of this sub-concept would include protecting and aggregating devices to culture planctonic organisms including larval stages of fish etc. or provide artificial reefs to increase fish populations and could involve stock enhancement and feeding of the existing fish. A special case is the combination of the green energy components with algae production. Here, the open ocean space in the vicinity of the concepts is used rather than the platform structure itself. The development of a plankton culture module would take advantage of the infrastructure that the platform offers, such as energy supply, shelter, anchoring, logistics for operation and maintenance, transportation or the artificial upwelling from the OTEC plant.

\section{B.3 Leisure components}

As accommodation facilities will be available the present concept has the capacity to host a significant number of people and therefore could be available (controlled and following strict security measures) as: (i) Specialized operational basis for high level training in offshore professional diving and open sea equipment operators extrapolating the operational platform infrastructures and the accommodation services. (ii) Equipment testing and validation for offshore technologies \& applications (including sensors, communications, surveillance equipment, spare parts and devises. Targeted to developers, constructors and final users; customers, due to consolidate relevant costs in real environmental extreme conditions taking advantage of existing platform infrastructure and expertise personnel that employed for platform operation.

\section{Leisure Island Concept}

This floating platform is moored in relatively shallow water closer to the shore which allows for the entire structure to be tugged to different sites. It has a modular structure with the following modules: water sports, a small marina, a diving facility, and an aqua farming structure. These modules can depending on the seasons - be moved separately to different locations. The main users of the facility will be the tourists staying in the hotels onshore. This will involve the use of a transfer which will stop at the leisure island for several hours including visits to the restaurants.

Since the beginning of the leisure concept task, with the preliminary study of the state of the art, the definition of the possible activities to be carried out on the floating platform and the facilities needed to provide services to the customers has progressed. The meetings held at PLOCAN (Oceanic Platform of the Canary Islands) with local stakeholders have been very fruitful with a lot of proposals and the idea of the platform location to be close to the coast line was a very important subject to be considered for the viability of the leisure concept of the TROPOS project.

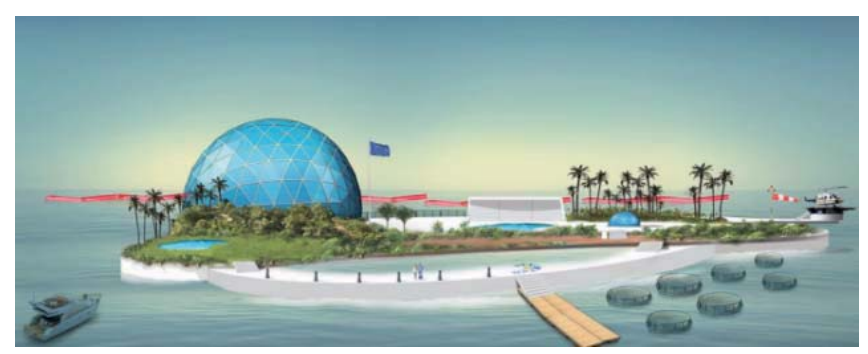

Fig. 6. Leisure Island Concept Representation [9]

The fact that there is no leisure offer of this kind on the high seas and therefore far away from the coasts means that targeted regions have the opportunity to begin a totally new project within the touristic sector. In such a project the leisure and scientific activities will unite with the rest of the activities that will take place on the platform such as taking advantage of the renewable energies with the aim to create clean energy, the transport to allow easier access of people and goods and finally the production of food by means of aquaculture. 
By undertaking this project, an important boost to the local economy will be given, not only because of the creation of jobs derived from the multiple activities that will take place, but also because of the opportunity that this "know how" will be exported to other countries.

\section{C.1 Transport components}

This floating platform is moored in relatively shallow water closer to the shore which allows for the entire structure to be tugged to different sites. It has a modular structure with following modules: Watersports, a small marina, a swimming and diving facility and aquafarming. These modules can depending on the seasons - be moved separately to different locations. The main users of the facility will be the tourists staying in the hotels onshore. They will visit the site on a daily basis for fun as well as for training courses etc. This will involve the use of a transfer service in the type of ferries as well as boat cruises around the main islands which stop at the leisure island for several hours including visits to the restaurants. The infrastructure allows for large cruise ships to anchor in the proximity and provide access to the site for these tourists.

\section{C.2 Energy components}

The floating island is mainly self-sufficient with regard to its energy demand in particular for electrical power, air conditioning within the buildings and hot water. To achieve this it extensively uses solar energy (PV and solar-thermal) which is integrated into the architecture of the island. In addition many small wind turbines are distributed over the structure or are integrated into the architecture as well. All other uses including drinking water and waste water treatment, supply of energy to the marina and aquafarming facilities.

\section{$\underline{\text { C.3 Aquaculture components }}$}

The aquaculture component will be developed in a compatible way with the leisure activities described above, aiming to cover four activities:

- To provide fish for the restaurants on the island.

- To develop a "fishing component" for the visitors.

- To develop artificial reefs for snorkelling - diving.

- To handle part of the produced organic waste.

The "fishing component" comprises of an appropriate cage located close to the main touristic activity where fish (at commercial size) will be available for "angling" by the visitors.

The artificial reefs will be developed either on the bottom or at medium depths and could be managed either after populating them with cultured individuals or by supporting the naturally populated individuals with additional feeding.

Finally, due to a large number of daily visitors and their supply significant amounts of waste and waste water are generated. The organic part of the waste is treated for use in a nearby aquafarming installation - which is designed not to compromise the water quality of the sensitive near shore environment as well as the diving and swimming areas (details to be discussed).

\section{C.4 Leisure components}

Since the entire installation is focusing on leisure activities it offers a wide range of activities from swimming, diving, fishing, sailing and all kinds of water sports, kiting, etc. At the same time the overall concept due to its renewable energy supply and integrated waste treatment builds on the concept of sustainable, responsible and high-quality tourism. This also includes educational aspects on the marine life etc.

The facility has restaurants but due to its proximity to the shore and efficient transfer service overnight accommodation for tourists is limited. Accommodation is mainly oriented to a small number of people such as service personnel for the operation and maintenance of the facilities.

\section{SYNERGIES AMONG COMPONENTS}

Once studied, the three proposed concepts are adequate to find out the synergies among the four presented components: Transport, Energy, Aquaculture and Leisure for each one of the concepts.

In the case of the Industrial Complex, the TROPOS Project Deliverable 2.1 shows some of the key aspects of the compatibility assessment for the TEA components. The most critical are highlighted:

- There is a potential synergy among all Transport categories and all the Energy component categories, depending on whether the platform has an selfsufficient energy supply or not.

- Wind energy, in the case of electricity production, may be conflicting with all Transport categories due to restricted access to wind farm areas.

- Wave energy may impair several other uses or components if the electricity was to be injected into the electrical grid, as this purpose implies the need of a considerably high wave power level. However, if the energy extraction feeds the platform or modules, the wave energy should be significantly more compatible with other uses, since it does not imply this need.

- OTEC is regarded as having no real incompatibilities with other TEAL components. It should not rule out any other considered TEAL component on the platform.

Regarding the Green\& Blue Concept aquaculture is an activity that is potentially compatible with most of the other uses considered for the platform and may create synergies with them. The effect of aquaculture itself on the other activities and on the environment may be limited provided that strict environmental management of all effluents is applied both at platform level and at module level. The platform activities ought to be distributed and located in such a manner that they will have no significant detrimental effects on each other. In the case of biofuel production from algae there is a clear synergy with energy carriers transport. 
Finally, the Leisure Island Concept is combined with the other TEA components were aspects such as water resources, climate change, pressure on biodiversity, environmental footprint, reduced greenhouse gas emissions are a big challenge for the future to mitigate the effects of the fossil fuels. Therefore, the TROPOS project will have a notorious impact at the socioeconomic level able to start changing mentalities thanks to its sustainable model.

\section{CONCLUSIONS}

The three different platform configurations (concepts) presented in this paper form an initial basis in the FP7 funded TROPOS Project to begin the engineering design of the platform, which will consider other project inputs related to the constraints and the resource assessment for the project components: Transport, Energy, Aquaculture and Leisure.

\section{REFERENCES}

[1] N. Harito, Introduction to the Analysis and Design of Offshore Structures - An Overview, EJSE (Electronic Journal of Structural Engineering) Special Issue: Loading on Structures, 2007.

[2] Proposal for a Directive of the European Parliament and of the Council establishing a framework for maritime spatial planning and integrated coastal management, Council of the European Union, COM (2013) 133, 2013.

[3] E. Quevedo, E. Delory, A. Castro, O. Llinás and J. Hernández for the TROPOS Consortium, Modular multi-purpose offshore platforms, the TROPOS project approach, International Conference on Ocean Energy (ICOE), 2012

[4] FP7 funded H2OCEAN Project (2012) [Online] Available:

http://www.h2ocean-project.eu

[5] FP7 funded MERMAID Project (2012) [Online] Available: http://www.mermaidproject.eu

[6] TROPOS Project (2012) [Online] Available: http://www.troposplatform.eu

[7] Deep Sea Monsters, Der Spiegel, 2010.

[8] Functional components for the TROPOS multiuse platform, The TROPOS Consortium, 2011.

[9] Fernando Montecruz for the TROPOS Project, 2013

[10] Yoza, Brandon A.; Nihous, Gérard C.; Takahashi, Patrick. K.; Golmen, Lars G.; War, Jan C.; Otsuka, Koji; Ouchi, Kazuyuki; Masutani, Stephen M., Deep Ocean Water Resources in the $21^{\text {st }}$ Century, Marine Technology Society Journal, Volume 44, Number 3, pp. 80-87(8), May/June 2010.

[11] TROPOS Project Deliverables, 2013 [Online]. Available: http://www.troposplatform.eu/Media2/Project-Deliverables 